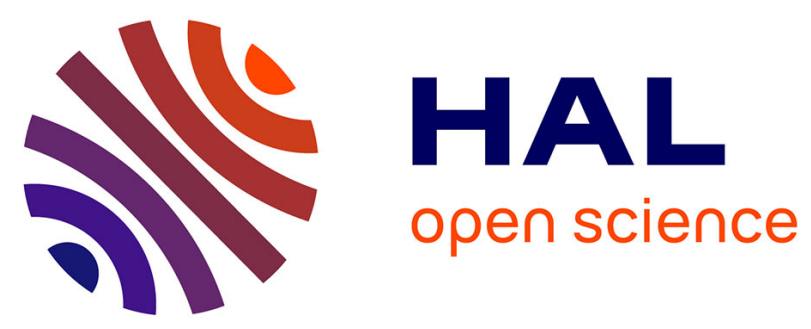

\title{
Un capteur détectant le fassage de bulles dans une couche fluidisée
}

H. Masson

\section{To cite this version:}

H. Masson. Un capteur détectant le fassage de bulles dans une couche fluidisée. Revue de Physique Appliquée, 1978, 13 (2), pp.93-94. 10.1051/rphysap:0197800130209300 . jpa-00244427

\section{HAL Id: jpa-00244427 https://hal.science/jpa-00244427}

Submitted on 1 Jan 1978

HAL is a multi-disciplinary open access archive for the deposit and dissemination of scientific research documents, whether they are published or not. The documents may come from teaching and research institutions in France or abroad, or from public or private research centers.
L'archive ouverte pluridisciplinaire HAL, est destinée au dépôt et à la diffusion de documents scientifiques de niveau recherche, publiés ou non, émanant des établissements d'enseignement et de recherche français ou étrangers, des laboratoires publics ou privés. 
Classification Physics Abstracts

07.90

\title{
UN CAPTEUR DÉTECTANT LE PASSAGE DE BULLES DANS UNE COUCHE FLUIDISÉE
}

\author{
H. MASSON
}

Service de Génie Chimique, Université libre de Bruxelles, 1050 Bruxelles, Belgique

(Reçu le 26 septembre 1977, accepté le 7 novembre 1977)

\begin{abstract}
Résumé. - La présente note décrit une sonde lumineuse permettant la caractérisation des propriétés des bulles dans un système biphasique.

L'interprétation repose sur la construction des histogrammes de trois temps caractéristiques.

Abstract. - The present note describes a light probe enable to characterize the bubble properties in a two phase system.

The interpretation is made using the histograms of three characteristic times.
\end{abstract}

Les couches fluidisées sont des appareils largement utilisés à l'échelle industrielle. Leur principe est basé sur la mise en suspension de fines particules solides par un courant gazeux ascendant.

Ces appareils se caractérisent par la propagation rapide de trous, appelés communément bulles, à travers la suspension.

La mise au point d'un modèle mathématique réaliste de lit fluidisé nécessite la description des propriétés des bulles.

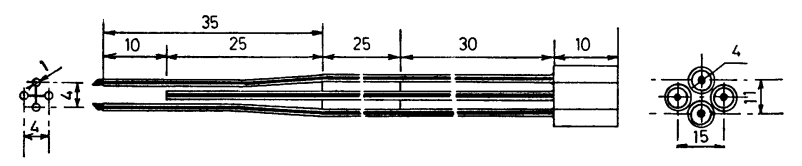

FIG. 1. - Schéma de la double sonde lumineuse.

[Double light probe.].

Nous avons développé un capteur à cet effet. Le schéma de principe est illustré à la figure 1. Le capteur comprend deux niveaux de mesure, distants de $1 \mathrm{~cm}$. A chaque niveau, une source de lumière alimente une fibre optique émettrice placée dans une seringue hypodermique pour assurer la rigidité. Une fibre réceptrice est disposée de façon similaire à $4 \mathrm{~mm}$ de l'émettrice. Elle est reliée à une photodiode dont le courant traverse un amplificateur opérationnel.

L'intensité de la lumière transmise varie selon la présence ou l'absence de bulles au point de mesure.

Une analyse d'amplitude (Fig. 2) montre qu'il est possible de distinguer les pics dus aux bulles de ceux résultant de fluctuations de la porosité de la suspension
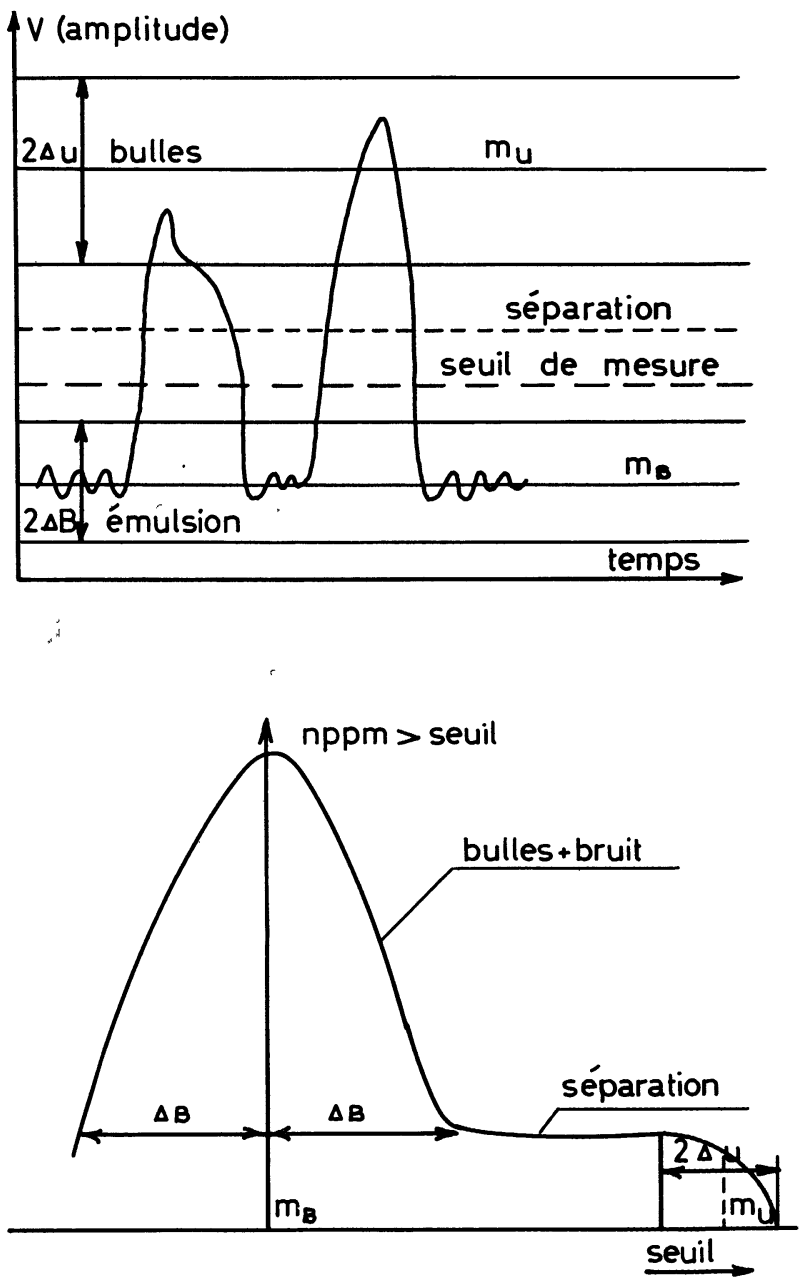

Fig. 2. - Principe de l'analyse d'amplitude.

[Principle of the amplitude analyse.] 
compte tenu du rapport des dimensions de la sonde à celles des particules utilisées.

L'analyse des signaux se fait en considérant trois temps caractéristiques (Fig. 3) :

$t_{1}=$ le temps de passage d'une bulle au niveau inférieur ;

$t_{2}=$ le décalage entre les signaux des deux niveaux;

$t_{3}=$ l'intervalle de temps entre deux bulles successives.

Ces temps sont largement distribués.

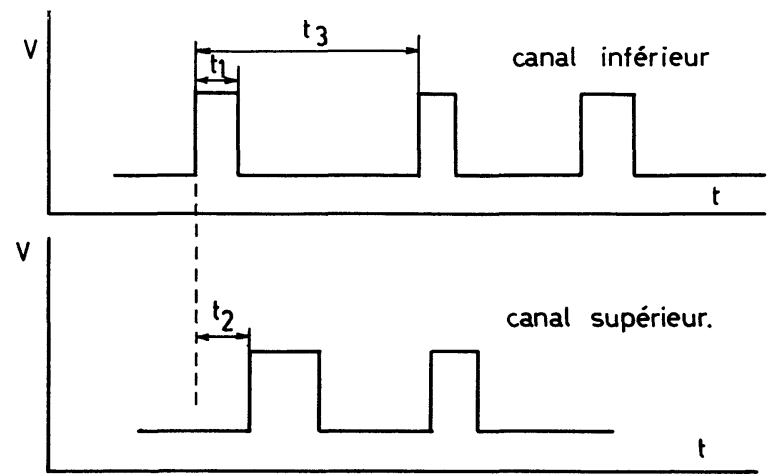

FIG. 3. - Définition des trois temps caractéristiques.

[Definition of the three characteristic times.]
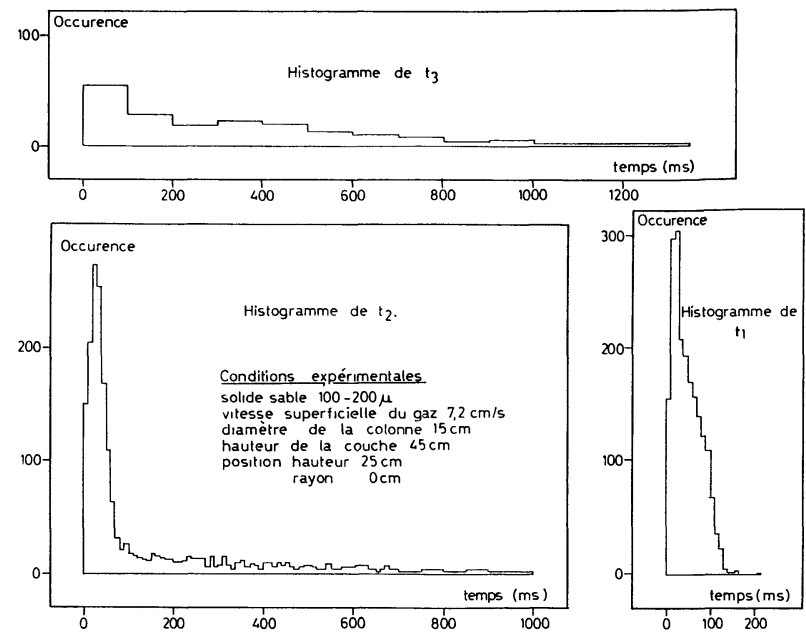

FIG. 4. - Exemple d'histogramme.

[Histograms of the three times.]

En utilisant un compteur digital relié on line à un ordinateur, nous avons construit les histogrammes des trois temps.

La figure 4 représente, à titre d'exemple, les histogrammes relevés en un point de la couche.

Les moyennes arithmétiques $\bar{t}_{1}, \bar{t}_{3}$ et la moyenne harmonique $\bar{t}_{2}^{\mathrm{H}}$ permettent de définir quatre grandeurs qui ont un sens physique important :

$$
\begin{aligned}
& v_{\mathrm{B}}=\frac{1}{\bar{t}_{2}^{\mathrm{H}}}=\text { la vitesse des bulles }(\mathrm{cm} / \mathrm{s}) \\
& \iota_{\mathrm{B}}=v_{\mathrm{B}} \bar{t}_{1}=\frac{1 \times \bar{t}_{1}}{\bar{t}_{2}^{\mathrm{H}}} \text { la corde verticale moyenne des bulles }(\mathrm{cm}) \\
& \varepsilon_{\mathrm{B}}=\frac{\bar{t}_{1}}{\bar{t}_{3}}=\text { la fraction volumique de bulles }(0) \\
& q_{\mathrm{B}}^{\prime}=\varepsilon_{\mathrm{B}} v_{\mathrm{B}}=\frac{1 \times \bar{t}_{1}}{\bar{t}_{2}^{\mathrm{H}} \bar{t}_{3}} \text { le débit visible de bulles par unité de section }(\mathrm{cm} / \mathrm{s}) .
\end{aligned}
$$

Les dimensions de la sonde permettent de définir la valeur ponctuelle de ces quatre grandeurs.

La technique est aussi transposable aux systèmes gaz-liquide sans modifications, pour autant que le liquide ne soit pas incolore. 